\title{
Breakpoint mapping and complete analysis of meiotic segregation patterns in three men heterozygous for paracentric inversions
}

Samarth Bhatt ${ }^{1,2}$, Kamran Moradkhani ${ }^{3}$, Kristin Mrasek ${ }^{4}$, Jacques Puechberty ${ }^{3}$, Marina Manvelyan ${ }^{4,5}$, Friederike Hunstig ${ }^{4}$, Genevieve Lefort ${ }^{3}$, Anja Weise ${ }^{4}$, James Lespinasse ${ }^{6}$, Pierre Sarda ${ }^{3}$, Thomas Liehr ${ }^{3}$, Samir Hamamah ${ }^{1,2,7,8}$ and Franck Pellestor*,1,2,7,8

${ }^{1}$ CHU Montpellier, Institute for Research in Biotherapy, Hôpital Saint-Eloi, Montpellier, France; ${ }^{2}$ INSERM U847, Montpellier, France; ${ }^{3} \mathrm{CHU}$ Montpellier, Service de Génétique Médicale, Hôpital Arnaud de Villeuneuve, Montpellier, France; ${ }^{4}$ Institute of Human Genetics and Anthropology, Jena, Germany; ${ }^{5}$ Department of Genetics and Laboratory of Cytogenetics, State University, Yerevan, Armenia; ${ }^{6} \mathrm{CHU}$ Chambery, Laboratoire de Cytogénétique, Chambéry, France; ${ }^{7}$ Université Montpellier I, UFR de Médecine, Montpellier, France; ${ }^{8}$ CHU Montpellier, Unité Biologie Clinique d'AMP/

DPI, Hôpital Arnaud de Villeuneuve, Montpellier, France

Paracentric inversions (PAIs) are structural chromosomal rearrangements generally considered to be harmless. To date, only a few studies have been performed concerning the meiotic segregation of these rearrangements, using either the human-hamster fertilization system or fluorescence in situ hybridization (FISH) with centromeric or telomeric DNA probes. To improve the assessment of imbalances in PAI, we present a new strategy based on FISH assay using multiple bacterial artificial chromosome probes, which allow a precise localization of chromosome break points and the identification of all meiotic products in human sperm. Sperm samples of three cases with PAI were investigated: an inv(5)(q13.2q33.1), an $\operatorname{inv}(9)(q 21.2 q 34.13)$ and an inv(14)(q23.2q32.13). The frequencies of spermatozoa with inverted chromosomes were $44.7 \%$ in inv(5), $42.7 \%$ in inv(9) and $46.7 \%$ in inv(14). The global incidences of unbalanced complements were $9.7,12.6$ and $3.7 \%$ in $\operatorname{inv}(5)$, inv(9) and inv(14), respectively. This report is the first study providing a detailed description of meiotic segregation patterns in human sperm by using a sperm FISH approach. This study demonstrates that the detailed analysis of segregation in PAI may provide important data for both genetic analysis and counseling of inversion carriers.

European Journal of Human Genetics (2009) 17, 44-50; doi:10.1038/ejhg.2008.144; published online 6 August 2008

Keywords: paracentric inversion; meiotic segregation analysis; sperm FISH

\section{Introduction}

Paracentric inversions (PAIs) are chromosomal rearrangements that occur after two breaks in a chromosome arm

${ }^{*}$ Correspondence: Dr F Pellestor, Department of Medicine and Biology of Reproduction, Arnaud de Villeneuve hospital, CHRU Montpellier, 371, avenue du Doyen Gaston Giraud, 34295 Montpellier Cedex 5, France. Tel: + 33 467335952; Fax: + 33467336290 .

E-mail: f-pellestor@chu-montpellier.fr

Received 22 January 2008; revised 23 June 2008; accepted 2 July 2008; published online 6 August 2008 followed by rotation of the chromosomal segment through $180^{\circ}$ and reinsertion of the segment. The presence of PAI may be unnoticed as they do not alter the chromosome arm ratio. Consequently, it is suspected that a certain percentage of small PAI remains undetected. ${ }^{1}$ Estimates of frequency range from 0.1 to $0.5 \%^{2,3}$ and PAIs are generally considered to be harmless for the carrier. Theoretically, heterozygous carriers of a PAI cannot produce viable unbalanced progeny. During meiosis, the occurrence of one (or an uneven number of) crossing-over event(s) 
within the inversion loop of affected segments should produce one dicentric and one acentric recombinant chromosomes, both of which are considered to be lethal. However, several cases of viable recombinant offsprings are reported, ${ }^{3-6}$ and a variety of mechanisms was proposed for the meiotic formation of stable recombinant chromosomes with deletion and/or duplication, including breakage of dicentric recombinants, ${ }^{1}$ breakage and reunion of sister chromatids, ${ }^{7}$ unequal crossover ${ }^{8}$ and the abnormal process of U-loop recombination. ${ }^{9,10}$ Also, the occurrence of spontaneous abortion, recurrent miscarriage and infertility refute complete innocuousness of the parental PAI and suggests that recombination here could arise more frequently than suspected from liveborn data.

The incidence of viable recombinants has been estimated to be $3.8 \%{ }^{11}$ but this figure remains controversial because of ascertainment biases and incomplete data. ${ }^{12,13}$ The direct chromosomal analysis of gametes allows overcoming this lack of information. However, only very few segregation analysis studies were performed for PAI.

Cheng et al ${ }^{14}$ analyzed a PAI of chromosome 7 in human oocytes and found a classical inversion loop in $10 \%$ of cells. Sperm karyotyping using the human sperm/hamster oocyte method was performed on two PAIs, one with $\operatorname{inv}(7)(q 11 q 22)$ and the other with an $\operatorname{inv}(14)(q 24.1 q 32.1)$. Brown et $\mathrm{al}^{15}$ used the sperm typing technique for studying patterns of recombination in sperm from a heterozygous carrier of a PAI of chromosome 9. Both these procedures were labor-intensive and provided data on small numbers of spermatozoa (from 94 to 282). The fluorescence in situ hybridization (FISH) technique has offered a new approach for segregation study in human sperm. However, only four PAIs have been investigated to date using the sperm FISH procedure with centromeric and/or telomeric DNA probes. These analyses provided data on large samples of human sperm (from 496 to 8158 ), but they did not allow the in situ identification of all the meiotic segregates because of the incomplete hybridization patterns obtained with centromeric or telomeric DNA probes.

To precisely determine the segregation patterns of chromosomal inversions in human gametes, we developed a high-resolution mapping strategy, based on the use of bacterial artificial chromosome (BAC) clones spanning the chromosomal break points. In the present study, we report the first application of this in situ high-resolution procedure on human sperm samples, for the direct segregation analysis of an $\operatorname{inv}(5)(\mathrm{q} 13.2 \mathrm{q} 33.1)$, an $\operatorname{inv}(9)(\mathrm{q} 21.2 \mathrm{q} 34.13)$ and an $\operatorname{inv}(14)(\mathrm{q} 23.2 \mathrm{q} 32.13)$.

\section{Materials and methods}

Three otherwise healthy men heterozygous for PAI inv(5), $\operatorname{inv}(9)$ and inv(14) were studied by molecular cytogenetics. The three patients, aged 60, 44 and 30 years respectively,
Table 1 BAC clones used for segregation analysis

\begin{tabular}{llc}
\hline $\begin{array}{l}\text { Cytogenetic } \\
\text { region }\end{array}$ & BAC/PAC clones & \multicolumn{1}{c}{ Position } \\
\hline 5q13.3 & RP11-114B1 & $74827703-74992978$ \\
& RP11-9N21 & $74962159-75118483$ \\
& RP11-179H5 & $74998605-75170876$ \\
& RP11-205H11 & $75138142-75273622$ \\
$5 q 33.1$ & RP11-263K13 & $150080197-150239374$ \\
& RP11-12A4 & $150265542-150418860$ \\
& RP11-96P14 & $150474047-150660861$ \\
$9 q 21.2$ & RP11-267G14 & $79046423-79213265$ \\
& RP11-466A17 & $79213269-79372174$ \\
& RP11-844C21 & $79301878-79514928$ \\
& RP11-235F20 & $132993351-133152079$ \\
& RP11-105C7 & $132816295-132908901$ \\
$9 q 34.13$ & RP11-544A12 & $132955072-133152093$ \\
& RP11-643E14 & $133146456-133324218$ \\
$14 q 23.2$ & RP11-676P5 & $63629431-63768477$ \\
& RP11-712C19 & $63768477-63912834$ \\
$14 q 32.13$ & RP11-262P9 & $93946936-94127939$ \\
& RP11-986E7 & $94090988-94303514$ \\
& RP11-179A9 & $94303515-94457888$ \\
\hline
\end{tabular}

were all ascertained through a fertility workup. All of them displayed normal sexual development and normal semen parameters (sperm count $>40$ million $/ \mathrm{ml}$; $>50 \%$ normal morphology and $>50 \%$ progressive motility). The three subjects were informed of the investigations planned, and they signed a consent form before participation in this study, which was approved by the ethical board of Montpellier University Hospital.

Initial cytogenetic studies of inv(5) and inv(14) were carried out on peripheral blood lymphocytes from the inversion carriers, using standard cytogenetic procedures for R- and G-banding. The chromosomal preparations from both the normal subjects and the inversion carriers were used for testing BAC probes and optimizing the in situ hybridization conditions. The selection of BAC clones and their preparation were performed as previously described. ${ }^{16}$ The breakpoint spanning BACs (Table 1) were used in sperm FISH along with a control BAC located outside the breakpoint regions. Sperm samples were processed according to the procedure of Pellestor et al. ${ }^{16}$ Briefly, each sample was washed three times in $1 \times$ PBS by centrifugation $(300 \mathrm{~g}$, $5 \mathrm{~min}$ ) and fixed for $1 \mathrm{~h}$ in fresh fixative (3:1 methanol: glacial acetic acid) at $-20^{\circ} \mathrm{C}$. The sperm suspension was then dropped onto clean microscope slides and air-dried. Slides were aged 2 days at room temperature before use for in situ chromosomal labeling. Before FISH procedure, the slides were immersed for $10 \mathrm{~min}$ in a pepsin solution $(50 \mathrm{ng} / \mathrm{ml}$ in $0.01 \mathrm{~N} \mathrm{HCl})$ prewarmed at $37^{\circ} \mathrm{C}$, washed for 2 min in $1 \times$ PBS and then dehydrated through an ethanol series (70, 90 and 100\%) and air-dried. The sperm nucleus 
decondensation and DNA denaturation was performed by slide incubation in $0.5 \mathrm{~N} \mathrm{NaOH}$ solution at room temperature for $8 \mathrm{~min}$, followed by a wash in $2 \times$ SSC, dehydration through an ethanol series and immersion in $70 \%$ formamide $/ 2 \times$ SSC solution $3 \mathrm{~min}$ at $73^{\circ} \mathrm{C}$. Finally, the slides were washed in $2 \times$ SSC, dehydrated through an ethanol series and air-dried.

The breakpoint characterization of inv(5) and inv(14) as well as the use of the breakpoint spanning BACs in sperm FISH analysis was published elsewhere. ${ }^{17}$ In the case of inv(9), only sperm sample was available. The BACs were selected according to the break points determined cytogenetically. A cocktail of continuous BAC clones was labeled with either digoxigenin-11-dUTP or biotin16-dUTP. Sperm FISH was performed to narrow down the breakpoint region. The BACs in contiguity spanning the break points (Table 1) were labeled and used as FISH probes.

\section{Results}

The labeling efficiency of BAC probes was determined on lymphocyte preparations and sperm preparations. The hybridization of the selected clones gave efficient results on both types of preparations with a satisfactory visualization of fluorescent signals as illustrated in Figure 1. For each probe mixture, the hybridization efficiency was estimated by scoring 50 metaphases and 100 sperm nuclei. The efficiency values ranged from 99.5 to $100 \%$ according to the probe set.

The size of the PAI in chromosome 5 was estimated as $41 \%$ of the total length of chromosome 5 . The BACs spanning the break points of $5 \mathrm{q} 13.3$ and $5 \mathrm{q} 33.1$ regions were RP11-179H5 and RP11-12A04, respectively. As shown in Figure 1a, the BACs RP11-179H5 and RP11-12A04 gave clear green and red signals, respectively, in normal spermatozoa.

The size of inversion in chromosome 9 was 39\% of the total chromosome length. The break points at $9 \mathrm{q} 21.2$ and 9q34.13 were narrowed down to BAC contiguities RP11267G14/RP11-466A17/RP11-844C21 and RP11-105C7/ RP11-235F20/RP11-544A12/RP11-643E14, respectively. In sperm harboring the inverted chromosome (Figure 1b), BAC probes gave specific patterns with either two yellow signals or two combined red and green signals, corresponding to the perfect or the incomplete colocation of BAC clones. On the other hand, sperm with a duplicated/ deficient chromosome gave either one red or one combined red/green signal, as illustrated in Figure 1c. Sperm nuclei with a dicentric chromosome displayed one red signal and one combined red/green signal (Figure 1d).

The size of the inverted segment in chromosome 14 was $29 \%$ of the total chromosome length. BACs spanning the
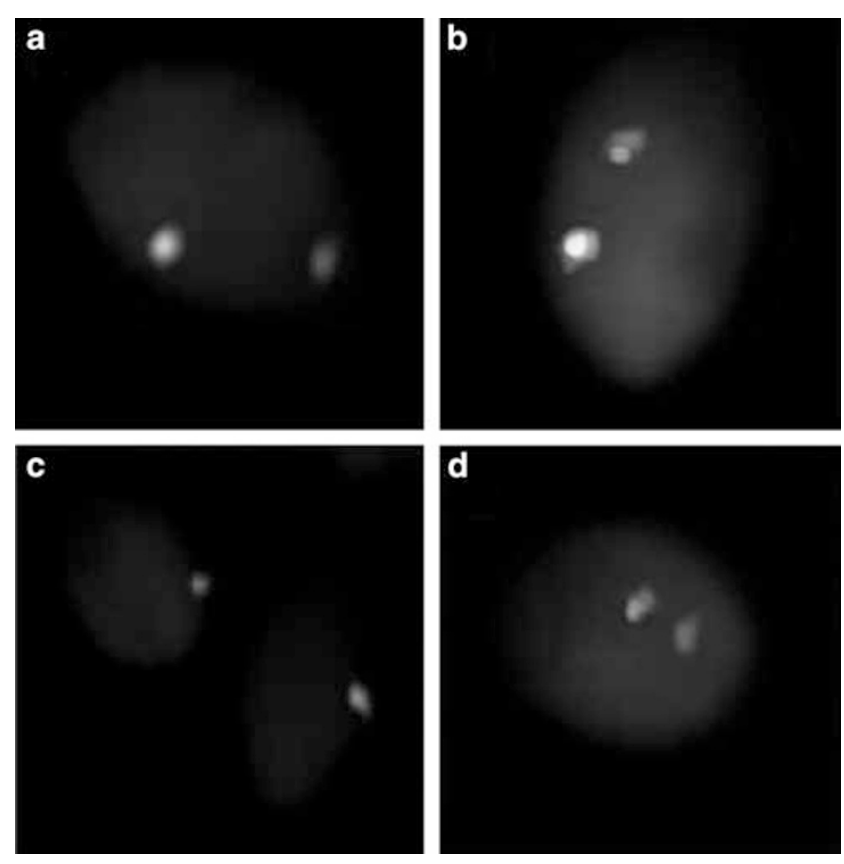

Figure 1 Meiotic diagrams and FISH signal patterns in spermatozoa using breakpoint-specific BAC clones. When using specific BAC clones spanning the break points, the in situ mixing of green and red signals gives yellow signals or a combined red/green signal on inverted or recombinant chromosomes, and thus allows the identification of each meiotic product, like (a) normal, (b) inverted, (c) duplicated/deficient, and (d) dicentric.

break points of $14 \mathrm{q} 23.2$ and $14 \mathrm{q} 32.13$ regions were RP11712C19 and RP11-986E7, respectively.

A total of 4807,1608 and 7670 sperm nuclei were evaluated for $\operatorname{inv}(5), \operatorname{inv}(9)$ and $\operatorname{inv}(14)$, respectively. The proportion of inverted chromosomes was $44.7 \%$ in inv(5), $42.7 \%$ in inv(9) and $46.7 \%$ in inv(14). The incidence of recombinants was $9.7 \%$ for $\operatorname{inv}(5), 12.6 \%$ for $\operatorname{inv}(9)$ and $3.7 \%$ for inv(14). Details of segregation analysis of these three inversions as well as of seven other PAIs previously analyzed are summarized in Table 2.

\section{Discussion}

The meiotic behavior of chromosomal PAI has hardly been explored in man. This may be linked to the low risk of imbalance generally attributed to chromosomal inversions. However, as indicated in Table 2, the technical difficulties inherent to the precise identification of all meiotic products in PAI also constitute important limitations for accurate segregation analysis in previous studies. Both sperm typing assay and sperm FISH with centromeric or telomeric probes were unable to identify all possible inversion segregants. Here, we demonstrate that BAC clones spanning the break points can be efficiently used as probes for precisely determining the segregation pattern 
of chromosomal inversions in human sperm. Of course, such an in situ mapping of break points is labor-intensive and takes longer than the use of commercial centromeric and subtelomeric probes. In addition, several authors have pointed out the lack of efficiency of subtelomeric probes for the identification of recombinant chromosomes in human sperm, and consequently the possible erroneous estimate of imbalance risk in male inversion carriers. ${ }^{23-25}$ However, by application of BAC probes, a complete and precise segregation analysis can be carried out, whereas previous sperm FISH studies have reported approximate rates of imbalances, with no details on dicentric and acentric recombinant products (Table 2). The present approach allows to distinguish all possible segregants from each recombination mechanism described in PAI (Figure 2), and thus to exclude the possibility of missing ones. These detailed data might contribute to a better exploration of the meiotic behavior of PAI, and they might also significantly improve the genetic counseling provided to inversion carriers.

Data on meiotic segregation in PAI heterozygous are limited for male carriers. Only 10 male carriers of PAI were studied to date. As indicated in Table 2, large variations are observed in incidences of recombinant gametes (from 0 to $12.6 \%)$. The variations in the number of sperm counted (from 94 to 8158) and inverted segment size (from 5 to $41 \%)$ are also observed. A direct correlation between the size of inverted segments and the production of recombinants in sperm has been postulated. ${ }^{26,27}$ This appears to be confirmed by our results obtained from large samples of segregation $(>1000)$, as an increase of recombination rates is observed in parallel with the increase in inverted segment size (Table 2).

To date, two PAIs of chromosome 14 with almost similar break points and inverted segment size have been reported. Nevertheless, no recombinant was observed in the study of Martin, ${ }^{19}$ whereas we found $3.7 \%$ chromosome 14 recombinants. The difference in the sample size in both studies could explain the lack of recombinants in the study of Martin. ${ }^{19}$ Only 120 sperm metaphases were scored, whereas in the present study, 7670 sperm nuclei were analyzed. The variations in recombinants in small and large sample sizes were also observed by Jaarola et al. ${ }^{28} \mathrm{~A}$ significant presence of recombinants in the present study of PAI of chromosome 14 could be due to the higher rates of recombination in shorter chromosomes than in the larger chromosomes. ${ }^{29}$ In the case of chromosome 14 , the recombination rate has been estimated as $1.36 \mathrm{cM} / \mathrm{Mb}$. Sun et $a l^{30}$ found a mean of 2.03 sites of meiotic exchange in the $\mathrm{q}$ arms of chromosome 14, which is also higher than that in the $\mathrm{q}$ arms of other chromosomes.

Our detailed segregation analysis of three PAIs shows that each inversion displays a significant rate of unbalanced gametes even with an inverted segment size less than $50 \%$ (Table 2), contrary to the assumption drawn 
a Meiotic diagrams

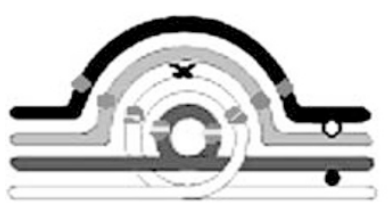

Signal patterns in spermatozoa

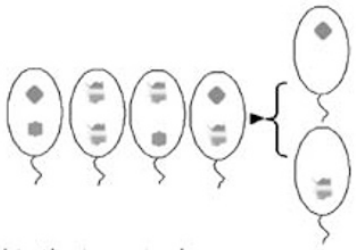

Crossing over within the inversion loop

b
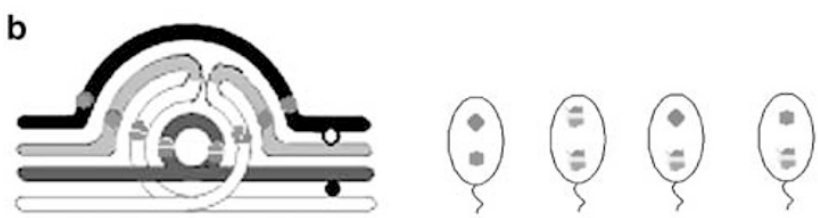

U-loop formation

c
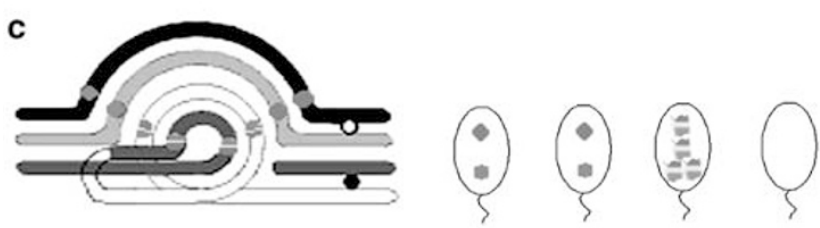

Breakage and reunion of sister chromatids

d
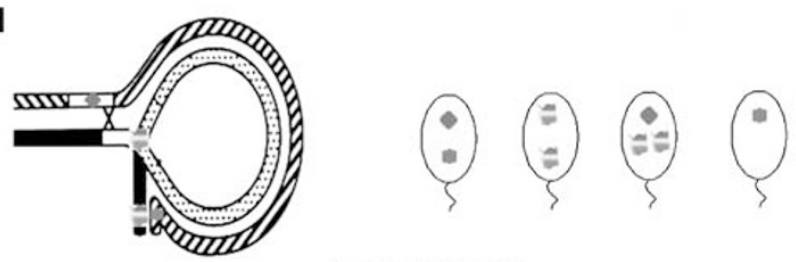

Unequal cross over

Figure 2 FISH labeling using BAC clones in sperm nuclei. (a) Sperm nucleus with a normal chromosome 5, displaying distinct green and red signals. (b) FISH results for BACs spanning the break points of the inverted chromosome 9 with one yellow and one collocated red/ green spots. (c) Duplicated/deficient chromosome with one red or one combined red/green signal. (d) Dicentric chromosome with one red and one collocated red/green signal.

from previous sperm studies that the production of recombinant gametes requires a minimum segment proportion of $50 \% .^{26,27}$ This needs to be confirmed on other inversions. However, this is consistent with the report of patients with recombinant chromosomes resulting from small inversions $(<45 \mathrm{Mb}$ and $<25 \%$ of total length of chromosome). ${ }^{31,32}$ The direct meaning of this observation in sperm could be that conventional sperm FISH assay using centromeric or telomeric DNA probes is definitively insufficient for a reliable segregation analysis of inversions because they can lead to underestimated rates of imbalances in sperm and then to erroneous genetic counseling of male inversion carriers.

Both chromosomes 5 and 14 are among the chromosomes most frequently implicated in PAI. ${ }^{1}$ The review of PAI studies has indicated that some particular chromo- somal regions could be more prone to breakage and recombination. Ashley ${ }^{33}$ proposed that in inversions, both loop formation and the potential for recombination occur only when both break points are located in G-light chromosomal bands. Of the 10 PAIs analyzed in sperm to date (Table 2), a majority of them displayed break points in G-light domains. Manvelyan et $a l^{34}$ also observed the preferential appearance of the break points in the G-light domains in chromosomal rearrangements. On chromosome 14, breakages in G-light bands 14q13, 14q24 and $14 \mathrm{q} 32$ appear to be more frequent. ${ }^{1}$ Indeed, there is some evidence that certain particular genomic features might facilitate or predispose to chromosomal rearrangements such as translocations or inversions. ${ }^{35}$ The sequences around the rearrangement break points are frequently enriched by low copy repeats (LCRs) predisposing to nonallelic homologous recombination (NAHR), as reported by Giorda $e t \mathrm{al}^{36}$ for the recurrent inv dup del(8p). Break points can be also colocalized with fragile sites, which promote genomic instability and double-strand breaks. The breakpoint 14q23.2 was found adjacent to the fragile site FRA14B. No LCRs or segmental duplications were found in the breakpoint regions of the PAI of chromosomes 5 and 14. This shows that for these two PAIs, LCRs were not involved in the formation of the inversion. Recently, Flores et $a l^{37}$ have identified in human chromosomes, several intrachromosomal identical reversed repeats of at least 400 nucleotides in length, which lead to chromosomal inversions by NAHR. These potential recombinogenic inverted sequences are distributed among all human chromosomes, suggesting that inversion rearrangements resulting from NAHR occur in a relatively high frequency in the human genome but preferentially on a nonvisible submicroscopic level. ${ }^{38,39}$ These genomic rearrangements might play a prominent role in human genetic disease. Our understanding of all these structural genomic features as well as the growing number of discovered individual variations and their chromosomal consequences is still limited. The genomic rearrangements are potentially reversible and thus they do not permanently compromise on either the structure or the dynamics of the genome, but some of these genomic variations could be more deleterious because of position effect, direct disruption of critical genes or interaction with chromatid packaging. There is still more to learn about the genome structural features predisposing some chromosomes to rearrangements.

For PAI, the standard statement has it that there is a negligible risk of producing viable unbalanced offspring. However, reports of patients with recombinant chromosomes and the identification of various alternate mechanisms for recombinant generation (U-loop recombination, breakage and reunion of sister chromatids) refute the complete harmlessness of PAI. The formation of a dicentric chromosome during meiosis should not be considered as a rare or a benign event. Our detailed segregation analysis 
indicates that in male carriers, the production of imbalances may be significant. In oogenesis, Koelher et al ${ }^{40}$ showed that segregation attempts in dicentrics could result in the premature loss of sister centromere cohesion and the segregation of an intact dicentric. Such meiotic behavior could explain the cases of viable stable dicentrics of maternal origin reported to date. ${ }^{3-6}$ Consequently, the production and the transmission of recombinant chromosomes through meiosis must be kept in mind when counseling carriers of PAI. In addition, efforts must be made to accurately identify all possible recombinants and distinguish between PAI and intrachromosomal insertion, which displays a significantly higher risk of genetic imbalance (from 15 to $30 \%$ ) than PAI. ${ }^{13}$ The in situ mapping of chromosomal break points by probe contigs, as presented herein, constitutes the first and efficient approach to avoid erroneous diagnosis. Combined with high-resolution genomic screening, this approach may yield new insights into the mechanisms involved in the generation of unbalanced rearrangements.

\section{Acknowledgements}

Supported in part by the IZKF Jena, DFG (436 ARM 17/11/06, LI 820/ 15-1), DAAD (A0704616/Ref326), Ferring Pharmaceuticals and Organon France. We thank the Mapping Core and Map Finishing groups of the Wellcome Trust Sanger Institute for initial clone supply and verification.

\section{References}

1 Madan K: Paracentric inversions: a review. Hum Genet 1995; 96 : 503-515.

2 Fryns J, Kleczkowska A, kubien E, van den Berghe H: Structural chromosome rearrangements in couples with recurrent fetal wastage: the leuven experience; in Sandberg A (ed): The Cytogenetics of Mammalian Autosomal Rearrangements. New York: Alan R Liss, 1988, Vol 8, pp 453-470.

3 Worsham MJ, Miller DA, Devries JM et al: A dicentric recombinant 9 derived from a paracentric inversion: phenotype, cytogenetics, and molecular analysis of centromeres. Am J Hum Genet 1989; 44: 115-123.

4 Mules EH, Stamberg J: Reproductive outcomes of paracentric inversion carriers; report of a liveborn dicentric recombinant and literature review. Hum Genet 1984; 67: 126-131.

5 Whiteford ML, Baird C, Kinmond S, Donaldson B, Davidson HR: A child with bisatellited, dicentric chromosome 15 arising from a maternal paracentric inversion of chromosome 15q. J Med Genet 2000; 37: E11.

6 Lefort G, Blanchet P, Belgrade N et al: Stable dicentric duplicationdeficiency chromosome 14 resulting from crossing-over within a maternal paracentric inversion. Am J Hum Genet 2002; 113: 333-338.

7 Phelan MC, Stevenson RE, Anderson Jr EV: Recombinant chromosome 9 possibly derived from breakage and reunion of sister chromatids within a paracentric inversion loop. Am J Med Genet 1993; 46: 304-308.

8 Yang SP, Bidichandani SI, Figuera LE et al: Molecular analysis of deletion (17)(p11.2p11.2) in a family segregating a $17 \mathrm{p}$ paracentric inversion: implications for carriers of paracentric inversions. Am J Hum Genet 1997; 60: 1184-1193.
9 Feldman GL, Weiss L, Phelan M, Shroer RJ, Van Dyke DL: Inverted duplication of $8 \mathrm{p}$ : ten new patients and review of the literature. Am J Med Genet 1993; 47: 482-486.

10 Mitchell JJ, Vekemans M, Luscombe S et al: U-type exchange in a paracentric inversion as a possible mechanism of origin of an inverted tandem duplication of chromosome 8. Am J Med Genet 1994; 49: 384-387.

11 Pettenati MJ, Rao PN, Phelan MC et al: Paracentric inversions in humans: a review of 446 paracentric inversions with presentation of 120 new cases. Am J Med Genet 1995; 55: 171-187.

12 Sutherland GR, Callen DF, Gardner RJM: Paracentric inversions do not normally generate monocentric recombinant chromosomes. Am J Med Genet 1995; 59: 390.

13 Madan K, Nieuwint AW: Reproductive risks for paracentric inversion heterozygotes: inversion or insertion? That is the question. Am J Med Genet 2002; 107: 340-343.

14 Cheng EY, Chen YJ, Disteche CM, Gartler SM: Analysis of a paracentric inversion in human oocytes: nonhomologous pairing in pachytene. Hum Genet 1999; 105: 191-196.

15 Brown GM, Leversha M, Hulten M, Ferguson-Smith MA, Affara NA, Furlong RA: Genetic analysis of meiotic recombination in humans by use of sperm typing: reduced recombination within a heterozygous paracentric inversion of chromosome 9q32-q34.3. Am J Hum Genet 1998; 62: 1484-1492.

16 Pellestor F, Andréo B, Puechberty J, Lefort G, Sarda P: Analysis of sperm aneuploidy by PRINS; in Pellestor F (ed): PRINS and in situ PCR protocols, second edn. Totowa: Humana Press, 2006, pp 49-60.

17 Bhatt S, Moradkhani K, Mrasek K et al: Breakpoint characterization: a new approach for segregation analysis of paracentric inversion in human sperm. Mol Hum Reprod 2007; 13: 751-756.

18 Martin RH: Sperm chromosome analysis in a man heterozygous for a paracentric inversion of chromosome 7 (q11q22). Hum Genet 1986; 73: 97-100.

19 Martin RH: Sperm chromosome analysis in a man heterozygous for a paracentric inversion of chromosome 14 (q24.1q32.1). Am J Hum Genet 1999; 64: 1480-1484.

20 Devine DH, Whitman-Elia G, Best RG, Edwards JG: Paternal paracentric inversion of chromosome 2: a possible association with recurrent pregnancy loss and infertility. J Assist Reprod Genet 2000; 17: 293-296

21 Anton E, Vidal F, Egozcue J, Blanco J: Genetic reproductive risk in inversion carriers. Fertil Steril 2006; 85: 661-666.

22 Vialard F, Delanete A, Clement P, Simon-Bouy B, Aubriot FX, Selva J: Sperm chromosome analysis in two cases of paracentric inversion. Fertil Steril 2007; 87: 418. e1-418. e5.

23 Anton E, Blanco J, Egozcue J, Vidal F: Risk assessment and segregation analysis in a pericentric inversion inv6p23q25 carrier using FISH on decondensed sperm nuclei. Cytogenet Genome Res 2002; 97: 149-154.

24 Mikhaail-Philips MM, McGillivray BC, Hamilton SJ et al: Unusual segregation products in sperm from a pericentric inversion 17 heterozygote. Hum Genet 2005; 117: 357-365.

25 Chantot-Bastaraud S, Ravel C, Berthaut I et al: Sperm-FISH analysis in a pericentric chromosome 1 inversion, 46, XY, inv (1) (p22q42), associated with infertility. Mol Hum Reprod 2007; 13: $55-59$.

26 Anton E, Blanco J, Egozcue J, Vidal F: Sperm studies in heterozygote inversion carriers: a review. Cytogenet Genome Res 2005; 111: 297-304.

27 Morel F, Laudier B, Guerif F et al: Meiotic segregation analysis in spermatozoa of pericentric inversion carriers using fluorescence in situ hybridization. Hum Reprod 2007; 22: 136-141.

28 Jaarola M, Martin Rh, Ashley T: Direct evidence for suppression of recombination within two pericentric inversions in humans: a new sperm FISH technique. Am J Hum Genet 1998; 63: $218-224$.

29 Kong A, Gudbjartsson DF, Sainz J, Jonsdottir GM, Gudjonsson SA, Richardsson B: A high-resolution recombination map of the human genome. Nat Genet 2002; 31: 241-247. 
30 Sun F, Oliver-Bonet M, Liehr T et al: Human male recombination maps for individual chromosomes. Am J Hum Genet 2004; 74: $521-531$.

31 Ciccone R, Mattina T, Giorda R et al: Inversion polymorphisms and non-contiguous terminal deletions: the cause and the (unpredicted) effect of our genome architecture. I Med Genet 2006; 43: e19.

32 South ST, Swensen JJ, Maxwell T, Rope A, Brothman AR, Chen Z: A new genomic mechanism leading to cri-du-chat syndrome. Am J Med Genet A 2006; 140: 2714-2720.

33 Ashley T: G-band position effects on meiotic synapsis and crossing over. Genetics 1988; 118: 307-317.

34 Manvelyan M, Shreyer I, Hols-Herpertz I et al: Forty-eight cases with infertility due to balanced chromosomal rearrangements: detailed molecular cytogenetic analysis of the 90 involved breakpoints. Int J Mol Med 2006; 19: 855-864.
35 Stankiewicz P, Lupski JR: Molecular-evolutionary mechanisms for genomic disorders. Curr Opin Genet Dev 2002; 12: $312-319$.

36 Giorda R, Ciccone R, Gimelli G et al: Two classes of low-copy repeats comediate a new recurrent rearrangement consisting of duplication at 8p23.1 and triplication at 8p23.2. Hum Mutat 2007; 28: 459-468.

37 Flores M, Morales L, Gonzaga-Jauregui C et al: Recurrent DNA inversion rearrangements in the human genome. Proc Natl Acad Sci USA 2007; 104: 6099-6106.

38 Feuk L, Carson AR, Scherer SW: Structural variation in the human genome. Nat Rev Genet 2006; 7: 85-97.

39 http://projects.tcag.ca/variation/.

40 Koehler KE, Millie EA, Cherry JP et al: Sex-specific differences in meiotic chromosome segregation revealed by dicentric bridge resolution in mice. Genetics 2002; 162: 1367-1379. 